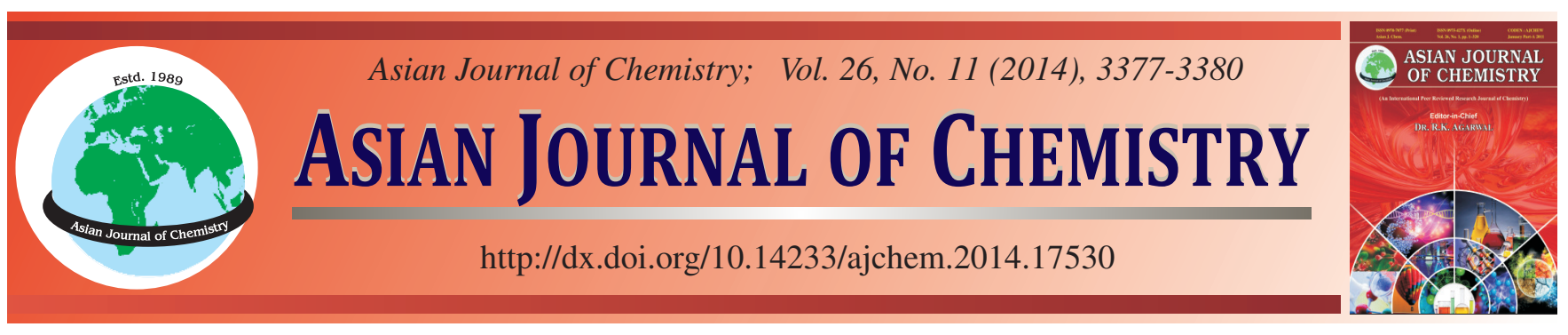

\title{
Multi-Scale Analysis Between Microstructure and Absorbing Performance of Thermal Sprayed Fe-(SiC-SiO$)_{2}$ Coatings
}

\author{
Xiaojing Yuan ${ }^{*}$, Xiaohu Chen, Xiaohui Cheng, Bailin Zha and Jie Xia
}

Xi'an Research Institute of Hi-Tech, Hongqing Town, Xi'an 710025, Shaanxi Province, P.R. China

*Corresponding author: Tel: +8629 84741370; E-mail: yxj2003@263.net

\begin{abstract}
Based on the characteristics of thermal sprayed particle, the fractal model was characterized the thermal sprayed composite absorber coatings and the mathematical model was established. The volume fraction of the absorber coatings was calculated with fractal dimension. Then, the coatings thickness and the interface can be analyzed for the microwave reflectivity coefficient. With the model, the effective permittivity and permeability of the nano-scales Fe-( $\left.\mathrm{SiC}_{-} \mathrm{SiO}_{2}\right)$ composite coating was calculated and measured. The result shows that when the mass fraction of the nano $\mathrm{SiC}$ is $28 \mathrm{wt} \%$, the coatings fractal dimension is 2.55081 and the volume fraction coefficient is 0.02423. The relationship between microstructure and performance of coatings were founded with the developed effective medium theory. The experiment result show that the coatings reflectivity coefficient of the coatings is distribute -2 to $-14 \mathrm{~dB}$.
\end{abstract}

Keywords: High velocity oxygen fuel, Absorber coatings, Mathematical model.

\section{INTRODUCTION}

The microwave absorbing performance of thermal sprayed coatings is a non-linearly coupled with microstructure effect existing in absorber coatings, which depends on coatings microstructure for certain spray process ${ }^{1}$. The establishment of a rigorous relationship between properties and microstructure of a coating is the key point which will lead to production of a desirable coating. Presently, several papers illustrate the important relationship between the microstructure and the coatings performance. The coating characteristics depend on the morphology, which can be designed and adjusted for special applications. Li and Ohmori ${ }^{2}$ reported that the Young's modulus perpendicular to the coating plane and microstructure of thermal sprayed coatings has been theoretically established using an idealized micro-structural model consisting of the stacking of lamellae a few micrometers thick and flat circular plate theory. Sahraoui et al. ${ }^{3}$ reported a methodology to describe the surface state of thermally sprayed coatings, which is mostly related to the process operating conditions and to the feedstock characteristics. They show that comparisons with conventional methodologies do not permit a direct correlation: the fractal methodology proves to be sensitive to the measurement scale and to the calculation protocol. $\mathrm{Yu}^{4}$ obtained a fractal description of effective dielectric coefficient of composite material using the tradition effective medium theory and the widely used fractal theory. A distinctive property of many materials (include the thermal sprayed coatings) is the presence of internal microstructure containing sets of characteristic lengths in a range of scale ${ }^{5}$.

High velocity oxygen fuel (HVOF) is one of the leading thermal spray techniques. It allows the fabrication of variety of coatings characterized by a low or intermediate melting point (mainly metals and polymers). The main advantage of high velocity oxygen fuel compared to other thermal spray techniques is the ability to accelerate the melted powder particles of the feedstock material at a relatively large velocity. The microstructure of coatings exhibits self-similar, moderately different and significantly different topographies. The fractal dimension offers a semiquantitative tool for comparative evaluation of the complexity, in terms of connectivity and irregularity, of the defects shown by the studied samples. An algorithm for calculating the fractal dimension is proposed.

In this paper, rigorous treatment for non-linearly coupled performance-microstructure effect with effective medium theory ${ }^{6}$, we use an analytical method for the non-linearly coupled performance-microstructure behaviour for high velocity oxygen fuel sprayed nano $\mathrm{Fe}-\left(\mathrm{SiC}-\mathrm{SiO}_{2}\right)$ composite coatings. This method gives insight into the sensitivity of the effective microwave absorber coefficients of the coatings on the fractal microstructure. 


\section{Effective model for thermal sprayed coatings}

Fractal features of the thermal sprayed composite coating: Ordinarily, thermal sprayed coatings microstructure exhibits self-similar, moderately different and significantly different topographies. Images of this fractal structure (that were isotropic with increasing fractal dimension) were used to evaluate the classification accuracy of this method, for surface topographies exhibiting different complexity. So, in the multi scales micrograph for SEM of thermal sprayed coatings, the volume fraction of absorber phase can be calculated with the fractal dimension. In order to simplify the computing procedures, the absorber phase was assumed to be periodical, the particle was assumed to be a cube and the porosity was assumed to be harmonic.

The pattern recognition by hybrid fractal method has been developed and applied to computer generated images of fractal surfaces and scanning electron microscope (SEM) images of thermal sprayed coatings microstructure. Images of fractal structure (that were isotropic with increasing fractal dimension) were used to evaluate the classification accuracy of the fractal method, for surface topographies exhibiting different complexity.

If the value of the averaged area of the solid phase for the different area-scales $\mathrm{L}$ in this region, section $\mathrm{S}$ can satisfy the expression

$$
\mathrm{S}(\mathrm{X}) \sim \mathrm{L}^{\mathrm{D}}
$$

And the volume fraction of absorber phase in the coatings can be calculated.

$$
\mathrm{f}_{\text {coa }}=\mathrm{C} \varsigma^{3-\mathrm{D}}
$$

here, $\mathrm{C}$ is constant which can be gained by eqn. $2 . \varsigma$ is the effective scale of the absorber particle in the coatings, which is $\zeta=\mathrm{r}_{\min } / \mathrm{r}_{\max }$ in the computing region.

Such behaviour can be observed when there are enough agglomerate particles to display the same repetitive internal structure over a much greater distance than the size of the objects forming the coatings agglomerate. The structure is composed of fractal semi-dilute objects with a size of $r$. The overall structure may be described as consisting of elementary particles of the order of the initial scale combines into fractal agglomerates. A three-dimensional coatings structure is then formed by these agglomerates.

Effective electro-magnetic model of thermal sprayed composite coatings: The electromagnetic properties (permittivity $(\varepsilon)$ and permeability $(\mu)$ ) of composites coatings were calculated with effective medium theory. To investigate the effect of electro-magnetic, we calculated general design charts by the relative permittivity. The mixture electro-magnetic of the absorber coating is expressed by Jylha and Sihvola ${ }^{7}$.

$$
\mathrm{K}_{\text {eff }}=\mathrm{K}+\frac{\sum_{\mathrm{i}=1}^{3} \mathrm{FK}_{\mathrm{a}}\left(\mathrm{K}_{\mathrm{s}}-\mathrm{K}\right) / 3\left[\mathrm{~K}+\mathrm{N}_{\mathrm{i}}\left(\mathrm{K}_{\mathrm{s}}-\mathrm{K}\right)\right]}{1-\sum_{\mathrm{i}=1}^{3} \mathrm{FK}\left(\mathrm{K}_{\mathrm{s}}-\mathrm{K}\right) / 3\left[\mathrm{~K}_{\mathrm{a}}+\mathrm{N}_{\mathrm{i}}\left(\mathrm{K}_{\mathrm{s}}-\mathrm{K}\right)\right]}
$$

where $\mathrm{K}$ and $\mathrm{K}_{\mathrm{s}}$ is the relative permittivity $(\varepsilon)$ or permeability $(\mu)$ of the host and second phase, $F$ is the volume fraction of the absorber phase, $N_{i}$ is the depolarizing factor and the $K_{a}$ is an apparent permittivity, which is not equaling to the background permittivity and any longer.
From the experiment and the numerical results, not only the thickness (d) and simple dielectric parameters of coatings $(\varepsilon, \mu)$ but also the mixture volume fraction of composite powder (F), interface characteristics (coatings-substrate, air-coatings) are important to the electromagnetic characteristics of composite coatings by the thermal spray technique.

As a result, the effective electro-magnetic parameters are affected by the absorber phase, host phase, volume fraction of the absorber in the composite coatings and the depolarizing factor which is particle shape factor. Then, exact behaviour of the electromagnetic wave at the surface and in the bulk of the material will critically depend on dielectric and magnetic properties of the thermal sprayed coatings which are represented by the complex permittivity $(\varepsilon)$ and complex permeability $(\mu)$. The voltage reflectivity of electromagnetic radiation, $\Gamma$ under normal wave incident at the surface of a single-layer material backed by a perfect conductor can be expressed by

$$
\Gamma=\frac{\sqrt{\mu / \varepsilon} \tan h(\gamma d)-1}{\sqrt{\mu / \varepsilon} \tan h(\gamma d)+1}
$$

And the power reflectivity, $\mathrm{R}(\mathrm{dB})$ is defined by

$$
\mathrm{R}=20 \lg |\Gamma|
$$

where, $\Gamma$ is a complex number, $|\Gamma|$ is the modulus of $\Gamma, \mathrm{d}$ is the thickness of the sample. The constant $\gamma$ is a complex propagation factor,

$$
\gamma=\mathrm{j} \frac{2 \pi \mathrm{f}}{\mathrm{c}_{0}} \sqrt{\mu \varepsilon}
$$

where $\mathrm{f}$ is the frequency, $\mathrm{c}_{0}$ is the propagating velocity of electromagnetic wave in vacuum.

\section{EXPERIMENTAL}

In order to improve particle flight performance in the process of thermal spraying, the nanometer $\mathrm{Fe}-\left(\mathrm{SiC}-\mathrm{SiO}_{2}\right)$ composite particle [Fe: $\left(\mathrm{SiC}_{-} \mathrm{SiO}_{2}\right)=0: 1.0(\mathrm{H} 1), 0.28: 0.72(\mathrm{H} 2)$, $0.75: 0.25(\mathrm{H} 3)$ and $0.85: 0.15(\mathrm{H} 4)]$ was agglomerated with mechanical spraying granulating method. The composite coatings were fabricated by high velocity oxygen fuel thermal spraying technique. The sample substrate is Fe material which is pre-treated with spraying sand.

The microstructure of powder and coatings are shown with JSM-6460 scanning electricity mirror (SEM) test observation, the phase structure is constituted with Rigaku $\mathrm{D} / \mathrm{C}$ max-3 auto $\mathrm{X}$-ray diffraction instrument; the quality of coatings is tested with the a dish medicine weighing scales instrument HCTP11 B5.

The microwave reflectivity coefficients of the coatings is tested with the space glint method, the blast-off source of the electromagnetic wave produces with HP8350B microwave generation machine, the frequency is $10 \mathrm{MHz} \sim 26.5 \mathrm{GHz}$, the HP8757C network analysis instrument, frequency 2-18 GHz. The size of sample is $22.86 \mathrm{~mm} \times 10.16 \mathrm{~mm} \times 2.00 \mathrm{~mm}$. The sample size of reflectivity is $180 \mathrm{~mm} \times 180 \mathrm{~mm} \times 1.0 \mathrm{~mm}$.

RESULTS AND DISCUSSION

Characteristics of nano $\mathrm{Fe}-\left(\mathrm{SiC}_{\mathrm{SiO}}\right)$ composite coatings: Fig. 1 shows the characteristics of the nano $\mathrm{Fe}-\left(\mathrm{SiC}-\mathrm{SiO}_{2}\right)$ particle particles. The agglomerate composite particle which 
was reunited by spraying technique is sphere, but the grain size is distributed at the some scales (Fig. 1(b)). Some nano $\mathrm{SiC}$ phase and non-crystal phase $\left(\mathrm{SiO}_{2}\right.$ glass phase) exist in the composite particles, which is shown in Fig. 1(c).
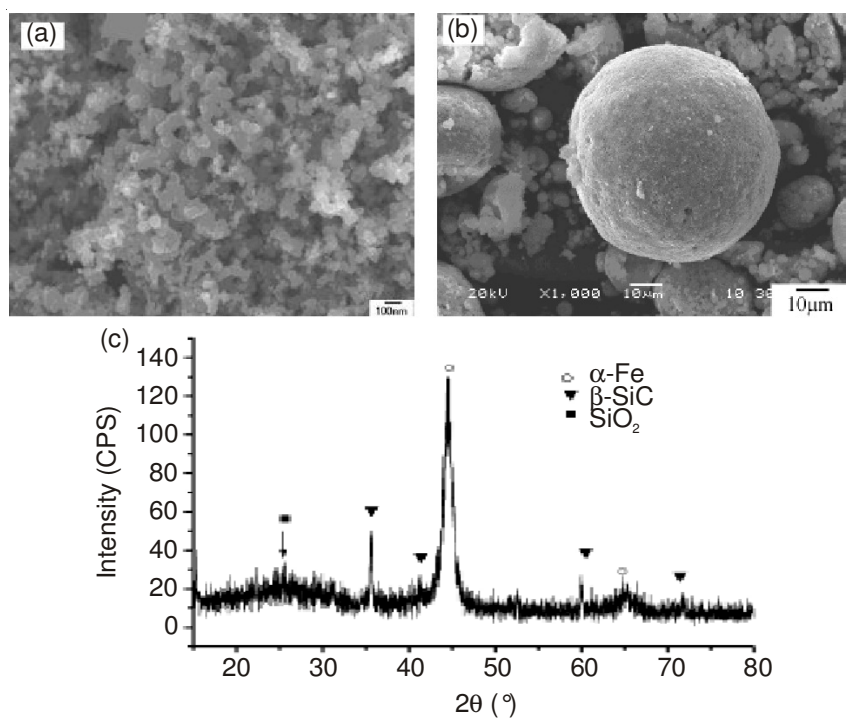

Fig. 1. Characteristics of nano Fe- $\left(\mathrm{SiC}-\mathrm{SiO}_{2}\right)$ agglomerat particle ((a) morphology of nanometer $\mathrm{SiC}$ particle; (b) and (c), morphology and XRD pattern of agglomerate sprayed particle

Fig. 2 shows the complex permittivity and permeability of the agglomerate composite particle, where the particle was added into the paraffin at $60 \mathrm{wt} \%$. The electromagnetic parameters were tested with the HP8350B microwave generation machine. It shows the real part $\left(\varepsilon^{\prime}\right)$ of permittivity is $15-45$ and the imaginary part ( $\left.\varepsilon^{\prime \prime}\right)$ of permittivity is $1-12$ in the composite particle. In order to measure the complex electro-magnetic parameters, the $\mathrm{Fe}-\left(\mathrm{SiC}-\mathrm{SiO}_{2}\right)$ agglomerate composite particles (mixing ratio is 70:30) mixed in paraffin, whose complex permittivity and complex permeability $\left(9.0-11.0\left(\varepsilon^{\prime}\right), 0.5-\right.$ 1.01( $\left.\varepsilon^{\prime \prime}\right), 1-2\left(\mu^{\prime}\right)$ and $\left.0.5-1.0\left(\mu^{\prime \prime}\right)\right)$, respectively (Fig. 2(c)). It shows that when the weight fraction of $\mathrm{Fe}$ is $75 \mathrm{wt} \%$, the microwave loss of ceramics $\mathrm{Fe}-\left(\mathrm{SiC}-\mathrm{SiO}_{2}\right)$ composite particle takes on imaginary dielectrics and permeability, where, $\varepsilon^{\prime \prime} / \varepsilon^{\prime}$ $=0.05-0.15$ and $\mu^{\prime \prime} / \mu^{\prime}=0.15-0.6$.

Fig. 3 shows the microstructure of the $\mathrm{Fe}-\left(\mathrm{SiC} / \mathrm{SiO}_{2}\right)$ composite coatings fabricated by high velocity oxygen fuel thermal spraying technology. Where, the glass phase was melted in the coatings and constructed to the stickiness in the high temperature (Fig. 3(a)). And, the nano-SiC was dispersed in the glass phase. At the same time, the $\mathrm{Fe}_{3} \mathrm{O}_{4}$ phase exists for the field of high velocity oxygen fuel thermal spraying process. From the XRD spectrum (Fig. 3(d)), the phase of the composite coatings is composed with the melted glass phase, $\mathrm{Fe}_{3} \mathrm{O}_{4}, \mathrm{Fe}_{2} \mathrm{O}_{3}$ and the SiC.

Fractal dimension of $\mathrm{Fe}-\left(\mathrm{SiC}-\mathrm{SiO}_{2}\right)$ composite absorber composite coatings: The calculation of the fractal dimension of the thermal sprayed $\mathrm{Fe}-\left(\mathrm{SiC} / \mathrm{SiO}_{2}\right)$ composite coatings is shown in Fig. 4, which is based on the fractal characteristic of EBSD image for the cross-section in the microstructure. The fractal dimension curve of $\mathrm{Fe}-\left(\mathrm{SiC}-\mathrm{SiO}_{2}\right)$ absorber coatings are shown in Fig. 4. From this figure, the fractal dimension is 2.55081 and he volume fraction coefficient is 0.02423 .
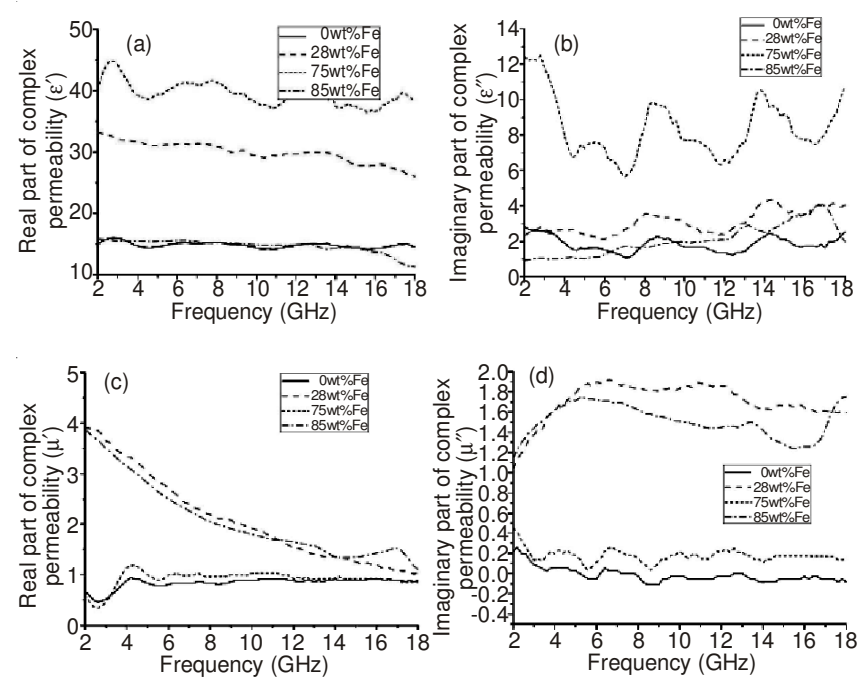

Fig. 2. Complex permittivity and permeability of nano $\mathrm{Fe}-\left(\mathrm{SiC}-\mathrm{SiO}_{2}\right)$ agglomerate particle: (a, b)real and imaginary part of complex permittivity; (c, d)real and imaginary part of complex permeability
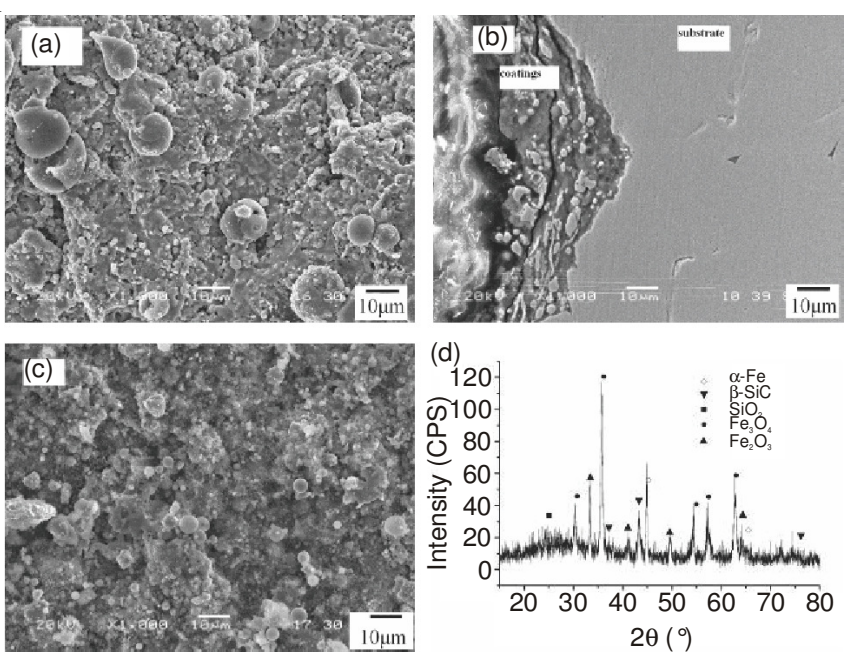

Fig. 3. Microstructure of Fe-( $\left.\mathrm{SiC}-\mathrm{SiO}_{2}\right)$ composite coatings: $((\mathrm{a}, \mathrm{b}, \mathrm{c})$ microstructure of the composite coatings, where the heretical section, cross-section and coatings surface, respectively and (d) XRD pattern of the composite coatings)

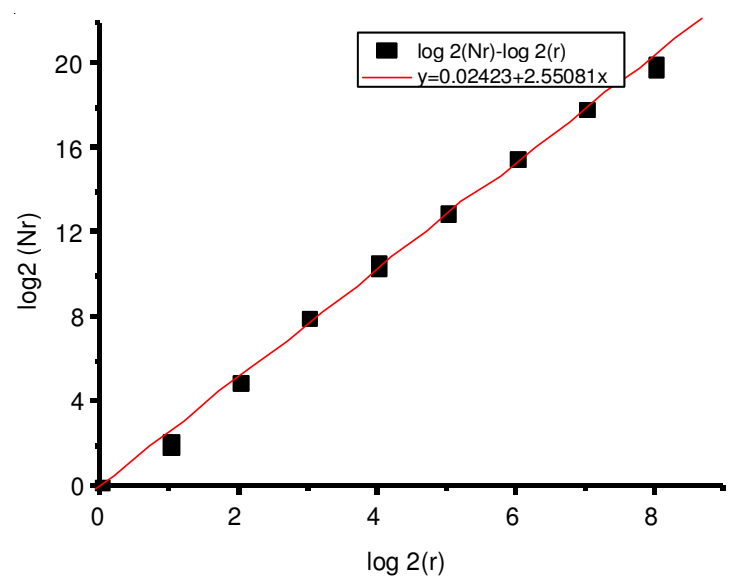

Fig. 4. Fractal dimensions of $\mathrm{Fe}-\left(\mathrm{SiC}-\mathrm{SiO}_{2}\right)$ composite coatings (28 wt \% $\mathrm{Fe})$

The multi-fractal diffraction of the absorber coating fabricated by high velocity oxygen fuel spraying process is shown in Fig. 5. It is noted that the Fe oxides extensively in 


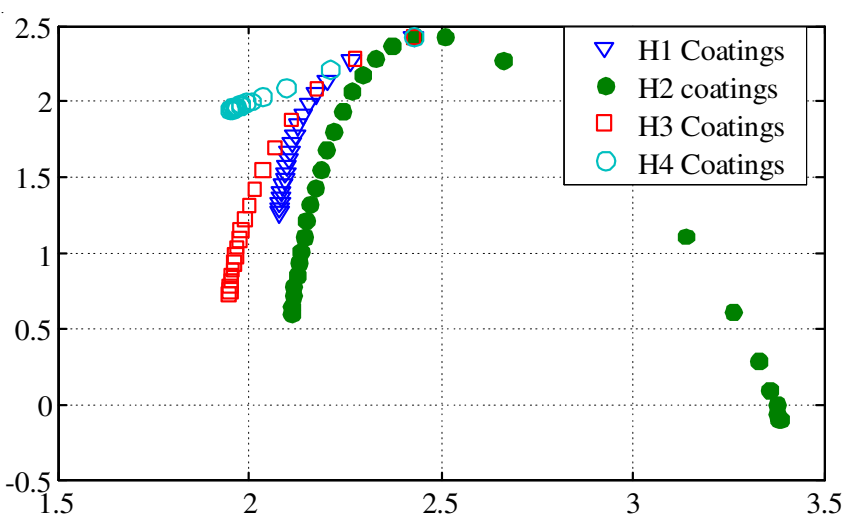

Fig. 5. Multi-fractal characteristics of Fe- $\left(\mathrm{SiC}-\mathrm{SiO}_{2}\right)$ composite coatings

the composite coatings during the building-up of the absorber coating. From the figure, the maximum of all of these coatings is approach to 2.5 . In these coatings, the $\Delta \alpha$ of $\mathrm{Fe}-\left(\mathrm{SiC}-\mathrm{SiO}_{2}\right)$ coatings is the largest and the $\alpha$ space of $\mathrm{H} 2$ coatings is the smallest, which is effected with the coatings' microstructure. At the same time, the experiment results show that the microwave reflectivity coefficient of $\mathrm{H} 2$ coatings is the lowest. From these result, the multi-fractal diffraction of the thermal sprayed coatings' microstructure is effective to the coatings performance.

The fractal model on the coatings' SEM microstructure shows that the composite absorber coatings have different fractal dimension and multi-fractal spectrum. The effective electromagnetic parameters can be calculated with the coatings' fractal dimension, absorber particles cluster electromagnetic parameters and the particle flatten ratio.

Evaluating of the microwave reflectivity efficient for thermal sprayed coatings: In the composite coatings, the complex permittivity of the $\mathrm{SiO}_{2}$ is 6.9. In the figures (Fig. 6), the microwave reflectivity of $\mathrm{H} 2$ coatings is optimal to others. Compared with calculation result, the shape of the particle in the coating can affect on the coatings effective electromagnetic parameters strongly.
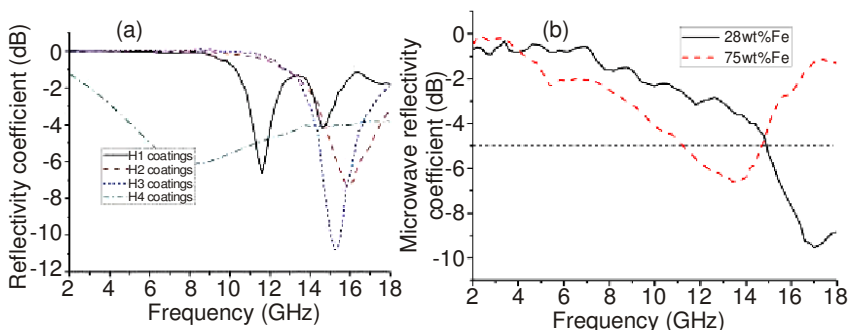

Fig. 6. Microwave reflectivity coefficient of thermal sprayed $\mathrm{Fe}-\left(\mathrm{SiC}-\mathrm{SiO}_{2}\right)$ composite coatings (a): calculated; (b) measured)
In order to obtain high temperature absorber coatings, the effects of easy-oxide metal particle $(\mathrm{Fe})$ on the nanoscales $\mathrm{SiC}$ glass matrix composite absorber coatings were also considered. The Fe particle is oxidized intensively in these coatings building-up process for the high temperature flame, so the coatings performance will be affected. The coatings fractal model can characterize to the thermal sprayed coatings electromagnetic performance. The evaluation between microstructure and performance of coatings were founded with the developed effective medium theory [Fig. 6(a)]. The simulation result shows that the developed effective medium theory can calculate the thermal sprayed coatings' effective electromagnetic. The experiment result shows that the coatings reflectivity coefficient of the coatings is distribute -2 to $-14 \mathrm{~dB}$.

\section{Conclusion}

In this paper, the classical effective medium theory was developed in the thermal sprayed coatings. Firstly, The thermal sprayed coatings possess the self-similar characteristics, so, the coatings characteristics are described with fractal dimension. In the nano $\mathrm{Fe}-\left(\mathrm{SiC}-\mathrm{SiO}_{2}\right)$ agglomerate composite absorber coating fabricated by high velocity oxygen fuel thermal spraying technique, the effective permittivity of the coating was researched with developed effective medium theory, where the absorber phase can be calculated with coating's fractal dimension.

The sprayed composite particle profile in the coatings was extracted with the fractal arithmetic. And the fractal dimension can be calculated for the volume fraction of the absorber coatings. As a matter of fact, the effective electromagnetic parameters model of thermal sprayed coatings is founded on the effective medium theory. Then, according to transmission line theory, the coatings thickness and the interface can be analyzed for the microwave reflectivity coefficient.

\section{REFERENCES}

1. X. Yuan, H. Wang, B. Zha, G. Hou and P. Hou, Surf. Coat. Technol., 201, 7130 (2007).

2. C.-J. Li and A. Ohmori, J. Thermal Spray Technol., 11, 365 (2002).

3. T. Sahraoui, S. Guessasma, M. Ali Jeridane and M. Hadji, Mater. Des., 31, 1431 (2010).

4. K.W. Yu, Phys. Rev. B, 49, 9989 (1994).

5. T.S. Choy and K.W. Yu, Phys. Rev. B, 52, 3341 (1995).

6. A.V. Dyskin, Int. J. Solids Struct., 42, 477 (2005).

7. L. Jylhä and A. Sihvola, J. Phys. D Appl. Phys., 40, 4966 (2007). 